\title{
Schizophrenia and atypical motor features in a case of progressive supranuclear palsy (the Steele-Richardson-Olszewski syndrome)
}

\author{
F. Ovsiew ${ }^{1}$ and J. Schneider ${ }^{2}$ \\ Departments of ${ }^{1 P s y c h i a t r y}$ and 2 Pathology, University of Chicago Hospitals \\ and The Pritzker School of Medicine, University of Chicago, 5841 S. Maryland, Chicago, \\ IL 60637, USA
}

\section{Correspondence to: F. Ovsiew at above address}

\begin{abstract}
Mental manifestations are characteristic of the syndrome described by Steele, Richardson, and Olszewski as progressive supranuclear palsy (SRO). Discussions emphasize cognitive aspects, namely the "subcortical dementia" for which the disease is prototypical, but personality change has been mentioned beginning with the earliest accounts. Psychosis has been virtually absent from neuropsychiatric descriptions, perhaps curiously so in view of the association between subcortical disease and delusions. We report here a case of autopsy-proven SRO in which a schizophrenia-like psychosis was a central feature.
\end{abstract}

Keywords: Motor features - Schizophrenia - Steele-Richardson-Olszewski syndrome - Supranuclear palsy

\section{CASE REPORT}

Mrs A was referred at age 64 because of self-endangerment by action on delusional beliefs. She was an accomplished professional woman formerly active in community affairs and the mother of three adult children.

There was no personal or family history of psychiatric illness, and she had been well (except for a remote hysterectomy) until 8 years earlier, when she complained of insomnia. Polysomnography revealed shortened REM latency, and depressive symptoms were treated with amitriptyline. She responded well, but micrographia and falling subsequently appeared. She began psychotherapy ostensibly to help with her adjustment to illness; however, the therapist confided to Mrs A's husband that she harbored delusional ideas about pregnancy. Markedly asymmetric dystonia developed, primarily involving the left arm, as well as drooling and progressive dysphagia requiring gastrostomy. Over the next several years, she proved unresponsive to anti-cholinergics, levodopa/carbidopa, and direct-acting dopamine agonists, and ultimately medication was stopped. The delusional belief persisted and became obvious to her husband and children.

Supranuclear gaze paresis appeared. She became progressively more inaccessible to communication, because of flexed posture, hypophonia, blepharospasm, and drooling. Nonetheless, her husband felt that she was mentally unimpaired except for the single delusional idea that she was pregnant. In October 1992, she was admitted to the Neuropsychiatry Service because on several occasions she had managed to arise from her wheelchair, don hat and coat, and head outdoors to meet her gynecologist so he could deliver her baby.

On examination, communication was difficult. She believed that she was pregnant, giving the date of conception as 16 October 1991. She said that she could tell she was pregnant because of a feeling of heaviness and fullness in her abdomen and a sensation in her head. She acknowledged that it was unusual to be pregnant for a full year and to become so after hysterectomy. She explained that she had grown a new uterus, having been selected to do so as compensation for her motor disability. She believed that the gynecologist was continuously present, as manifested by "clicks" that transmitted messages to her from him, and that he influenced (or at least read) her mind more directly. She indicated that he was at one point off to her right and "four feet off the floor", at another up and to the left; there was no characteristic spatial location of the auditory phenomena or of his presence. She believed that he loved her but offered no evidence for this beyond the above; she said that he was not the father of the child. She was happy about being pregnant and gave no indication of sadness or anxiety. She saw nothing abnormal in her beliefs but was aware that others did. She 
was well oriented and able to repeat a name and address and recall it after $3 \mathrm{~min}$. She used a number of lowfrequency words correctly in conversation and showed no anomia.

She was unable to perform voluntary upgaze or downgaze. The eyes moved laterally, albeit slowly, on pursuit but only erratically so on command ("that's hard to do"). She showed no medial movement of the eyes except on vergence. There was pronounced blepharospasm, worse on the left and markedly accentuated by any touch to the face, and grimacing of the lower face, with marked slowness of oral movement. The head was held flexed on the chest, though with effort she could bring it nearly upright; sensory tricks did not help. The left arm was held adducted, flexed at the elbow, and supinated. The fingers were hyperextended and occasionally showed athetoid movements. The right arm was more mobile but clumsy, with slowed finger movements, decremental finger tapping, and paratonic rigidity at the elbow. Rarely, the right arm assumed unusual postures, such as sticking straight up, but she was able to follow commands with that limb and denied that it "had a mind of its own". There was bilateral foot dystonia. She was unable to balance independently; supported, she took a few stiff-legged steps. Upper extremity tendon jerks were brisk, with spread; there was a left grasp reflex. Ankle jerks were absent. Plantar reflexes were extensor bilaterally. Sensation was much reduced to vibration only, at the ankles and knees; position sense was not evidently impaired. Two-point discrimination in the fingertips was symmetric.

Cerebral magnetic resonance imaging in 1989 was normal except for a few punctate white matter T2 hyperintensities. Neuropsychological examination in 1992 showed only mental slowing and difficulty with effortful retrieval, without cortical deficits.
Several weeks after discharge, her husband found her dead at home.

General autopsy did not disclose a specific cause of death. The brain weighed $1315 \mathrm{~g}$. Gross findings included brownish discoloration of both subthalamic nuclei, bilateral slight atrophy of the globus pallidus, and bilateral depigmentation of the substantia nigra. A small old cystic infarct was present in the right cerebellum. There was no cortical atrophy.

Formalin-fixed tissue blocks were taken from representative areas. Paraffin sections were stained with H\&E. Selected sections were also stained with Bodian, Bielschowsky, Prussian blue, and periodic acid Schiff. For electron microscopic study formalin-fixed tissue from the hippocampus and globus pallidus was embedded in epon and routinely stained.

Microscopic examination revealed marked neuronal loss with gliosis and numerous neurofibrillary tangles (NFTs; Fig. 1) in subcortical structures (Table I). There was severe loss of pigmented neurons in the substantia nigra and the locus ceruleus; excess pigment was free in the parenchyma and within macrophages in these areas, as well as in neostriatum, globus pallidus, and subthalamic nuclei. Special stains identified most of this pigment as iron and probable lipofuscin. All changes appeared bilaterally symmetric although quantitative analysis was not performed.

The hippocampus contained infrequent NFTs; senile plaques were absent from hippocampus and parahippocampus. Neither plaques nor tangles were found in the cortex. No neuronal loss or NFTs were seen in the entorhinal cortex. There were no inclusion bodies or achromatic neurons.

Electron microscopic examination of hippocampal sections with numerous pyramidal neurons showed no paired
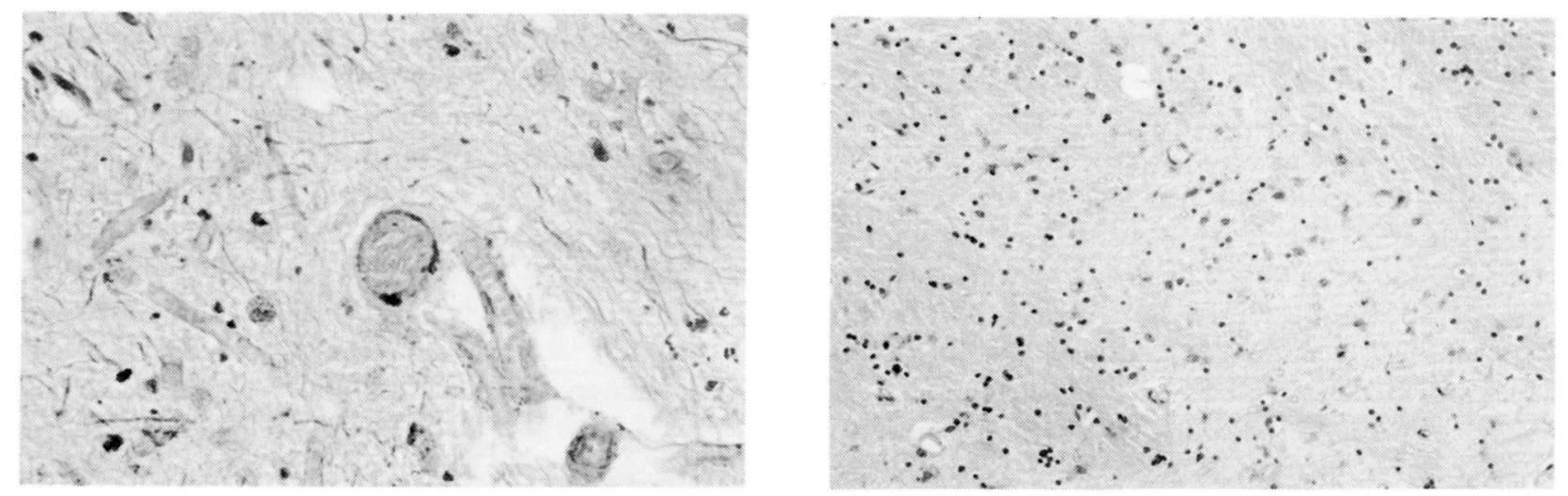

FIG. 1. (A) Substantia nigra, Bodian stain, original magnification $200 \times$. Neuron contains globose NFT, with melanin displaced peripherally. (B) Globus pallidus, H\&E, original magnification $80 \times$. Gliosis and neuronal loss are seen. 
TABLE I. Distribution of pathological findings

\begin{tabular}{lcc}
\hline Location & Gliosis/Neuronal Loss & NFTs \\
\hline Subthalamic nuclei & +++ & +++ \\
Globus pallidus & +++ & +++ \\
Caudate/putamen & 0 & + \\
Lateral septal nuclei & + & + \\
Substantia innominata & $0 /+$ & + \\
Hypothalamus & 0 & + \\
Colliculi & ++ & + \\
Periaqueductal gray & ++ & ++ \\
Locus ceruleus & + & ++ \\
Inferior olive & 0 & ++ \\
Hippocampus & 0 & + \\
Neocortex, thalamus, & & \\
dentate nuclei, pontine & & \\
nuclei, spinal cord & 0 & \\
\hline
\end{tabular}

Gliosis and neuronal loss: 0 , absent; + , mild; ++ , moderate; +++ , severe.

Neurofibrillary tangles (NFTs): 0 , absent; + , few; ++ , intermediate; +++ , many.

helical filaments. In the globus pallidus, neurons were difficult to identify because of autolysis and marked neuronal loss.

The pathologic findings were diagnostic of SRO.

\section{DISCUSSION}

The clinical picture of SRO has been thoroughly described (Ostergaard Kristensen, 1985; Lees, 1987; Jankovic et al., 1990). Gaze abnormality is the hallmark but may be delayed or even absent (Nuwer, 1981; Dubas et al., 1983; Davis et al., 1985). Internuclear ophthalmoplegia is a recognized pattern of gaze disorder in SRO (Mastaglia and Grainger, 1975). Falls, rigidity in a predominantly axial distribution, and dysarthria and other bulbar disturbances are the characteristic motor features. Our patient had two atypical motor features: flexion posture and asymmetric limb rigidity. The axial rigidity is characteristically in extension, but a flexed posture has been described in pathologically proven cases (Dubas et al., 1983). Dorsiflexion has been attributed to pathological involvement of the interstitial nucleus of Cajal (Fukushima-Kudo et al., 1987). We were unable to examine this structure. Asymmetric limb dystonia was seen in two patients by Léger $e t$ al. (1987) and in eight of 30 patients by Rafal and Friedman (1987). In the latter series, neuropathology was available in only one case. In that case, as in ours, no asymmetry was found pathologically. We considered a diagnosis of corticobasal degeneration (Thompson and Marsden, 1992), but the absence of cortical signs (myoclonus, cortical sensory or cognitive features) and characteristic histopathology excluded this diagnosis.

Albert et al. (1974) stimulated study of cognitive abnor- mality in SRO by recognizing a distinctive pattern of impairment, which they referred to as "subcortical dementia". This pattern had been previously delineated, with respect to the sequelae of encephalitis lethargica, under the designation "bradyphrenia" (Rogers, 1986). Our patient's mild cognitive disorder was characteristic of SRO. Impaired attention, slowed mentation, reduced verbal fluency, impersistence, and imitation and utilization behavior mark the cognitive impairment as related to frontal lobe dysfunction (Cambier et al., 1985; Lees, 1987). Though the anatomic pathology in SRO is subcortical, positron emission tomography demonstrates hypofunction of frontal cortex (D'Antona et al., 1985).

Expectably in a frontal lobe disorder, personality change is common. In the original nine case reports, Steele et al. (1964) used descriptors such as irritable, suspicious, arrogant, demanding, explosive, and facile; in several patients mental change at times dominated the clinical picture. Behrman et al. (1969) noted that "personality changes may be among the first symptoms of the disease". One of their patients showed a "paranoid attitude" but was not said to have been psychotic. Maher and Lees (1986) found personality change in 12 of 52 patients. Cambier $e t$ al. (1985) described prominent behavioral change in 10 patients; in five, these disturbances were "in the forefront at a certain point in the course" (our translation). The patients were apathetic as a rule, but jealousy (perhaps delusional), aggressive behavior, and depression with morbid preoccupation occurred. Personality change was not evident in our patient, but through much of her illness she was so disabled in movement and communication that personality change might have been obscured.

Psychotic phenomena are not explicitly mentioned in these series or others (Janati and Appel, 1984; Ostergaard Kristensen, 1985; Jankovic et al., 1990). In a neuropathological report cited by Steele et al. (1964) as a precursor of their own description, Verhaart (1958) said his patient showed paranoid delusions and loss of interest and underwent electroconvulsive therapy. Perkin et al. (1978) mentioned that a patient accused his wife of trying to poison him and was treated with anti-psychotic and anti-depressant drugs as well as with electroconvulsive therapy, presumably with a diagnosis of psychotic depression. Lee (1991) described a patient with the clinical diagnosis of SRO who showed persecutory delusions about his wife in the context of organic personality change. Takahashi et al. (1987) linked the occurrence of visual hallucinations and nocturnal delirium in a patient with autopsy-proven SRO to involvement of limbic structures by neurofibrillary tangles. Shimizu et al. (1990) described a patient with the clinical diagnosis of SRO who showed disturbed sleep architecture and delirious nocturnal behavior. Our patient appears to be the first described with a pathological diagnosis of SRO and a schizophrenic psychosis. 
The nosology of DSM-III-R (American Psychiatric Association, 1987) is not fully satisfactory to classify our patient. Diagnoses of organic delusional syndrome and organic hallucinosis can be made if we judge that the organic factor, i.e. SRO, was etiologic for her psychosis, a point discussed below. However, these terms carry little more meaning than to say that the patient had hallucinations and delusions. The ICD-10 (World Health Organization, 1992) discussion of organic delusional (schizophrenia-like) disorder indicates that "features suggestive of schizophrenia, such as bizarre delusions ... or hallucinations" (p.62) should lead to classification in this category. A DSM-III-R diagnosis of schizophrenia is excluded by the organic factor; onset after age 45 , though an exclusion criterion in DSM-III, is not a bar to the diagnosis in DSM-III-R. Because of the bizarre quality of our patient's delusional beliefs and the presence of passivity ideas, in the absence of a mood syndrome, we consider the illness schizophrenic in form. To some, the term "schizophrenia" implies the lack of an organic etiology, and thus a case such as ours must be designated schizophrenia-like or schizophreniform or by some other locution (Davison, 1983). We prefer to consider schizophrenia a behavioral syndrome that is occasionally of discernible organic etiology though most often idiopathic. From this point of view, our case would be called one of schizophrenia, with that syndromal diagnosis always necessitating a search for potential specific organic etiologies.

Was SRO etiologic in our patient's psychotic illness? If psychosis is as unusual in SRO as the above review suggests, perhaps the concurrence of SRO and schizophrenia is coincidental. Alternatively, why not accept her own diagnosis, that the ideas were restitutional for a psychologically painful loss of function?

The rarity of the association is as strongly against the reactive diagnosis as against the organic one. Further, the bizarre nature of the delusional ideas and the lack of persisting abnormal mood count against a reactive diagnosis, as does the onset of the psychosis early in the course, before disability was severe. Against the diagnosis of coincidental idiopathic schizophrenia are the absence of a family history of schizophrenia and the lack of pre-morbid personal psychopathology. Most late-onset psychoses are of organic origin, though idiopathic schizophrenia can begin in late life (Miller et al., 1991; Murray et al., 1992). An etiologic role for SRO is biologically plausible because of the association of complex delusions of organic origin with subcortical diseases (Bowman and Lewis, 1980; Cummings, 1985). A recent study found entorhinal cortical changes, associated with dementia, in SRO (Braak et al., 1992). These findings are of interest in regard to schizophrenia because of the evidence of architectonic abnormalities in entorhinal cortex in psychosis (Beckmann and Jakob, 1991). However, our (non-demented) patient lacked abnormalities, either architectonic or degenerative, in this region.

Only further psychiatric assessment of patients with SRO will reveal whether the concurrence reported here has general significance. Ideally, clinicopathological correlation may identify a specific local emphasis of degeneration or other factors associated with the development of psychosis.

\section{REFERENCES}

Albert ML, Feldman RG and Willis AL (1974) The 'subcortical dementia' of progressive supranuclear palsy. Journal of Neurology, Neurosurgery and Psychiatry, 37, 121-130.

American Psychiatric Association (1987) Diagnostic and Statistical Manual of Mental Disorders. American Psychiatric Association, Washington, DC.

Beckmann H and Jakob H (1991) Prenatal disturbances of nerve cell migration in the entorhinal region: a common vulnerability factor in functional psychoses? Journal of Neural Transmission, 84, 155-164.

Behrman S, Carroll JD, Janota I and Matthews WB (1969) Progressive supranuclear palsy. Brain, 92, 663-678.

Bowman M and Lewis MS (1980) Sites of subcortical damage in diseases which resemble schizophrenia. Neuropsychologia, 18, 597-601.

Braak H, Jellinger K, Braak E and Bohl J (1992) Allocortical neurofibrillary changes in progressive supranuclear palsy. Acta Neuropathologica (Berlin), 84, 478-483.

Cambier J, Masson M, Viader F et al. (1985) Le syndrome frontal de la paralysie supranucléaire progressive. Revue Neurologique, 141, 528-536.

Cummings JL (1985) Organic delusions: phenomenology, anatomical correlations, and review. British Journal of Psychiatry, 146, 184-197.

D'Antona R, Baron JC, Samson Y et al. (1985) Subcortical dementia: frontal cortex hypometabolism detected by positron tomography in patients with progressive supranuclear palsy. Brain, 108, 785-799.

Davis PH, Bergeron C and McLachlan DR (1985) Atypical presentation of progressive supranuclear palsy. Annals of Neurology, 17, 337-343.

Davison K (1983) Schizophrenia-like psychoses associated with organic cerebral disorders: a review. Psychiatric Developments, 1, 1-34.

Dubas F, Gray F and Escourelle R (1983) Maladie de SteeleRichardson-Olszewski sans ophthalmophégie. Six cas anatomo-clinique. Revue Neurologique, 139, 407-416.

Fukushima-Kudo J, Fukushima K and Tashiro K (1987) Rigidity and dorsiflexion of the neck in progressive supranuclear palsy and the interstitial nucleus of Cajal. Journal of Neurology, Neurosurgery and Psychiatry, 50, 1197-1203.

Janati A and Appel AR (1984) Psychiatric aspects of progressive supranuclear palsy. Journal of Nervous and Mental Disease, 172, 85-89.

Jankovic J, Friedman DI, Pirozzolo FJ and McCrary JA (1990) Progressive supranuclear palsy: motor, neurobehavioral, and neuro-ophthalmic findings. In Advances in Neurology, Vol. 55, Parkinson's Disease: Anatomy, Pathology, and Therapy (Eds MB Streifler, AD Korczyn, E Melamed and MBH Youdim), pp. 293-304. Raven Press, New York.

Lee $S$ (1991) The neuropsychiatric evolution of a case of pro- 
gressive supranuclear palsy. British Journal of Psychiatry, 158, 273-275.

Lees AJ (1987) The Steele-Richardson-Olszewski syndrome (progressive supranuclear palsy). Movement Disorders 2 (Eds CD Marsden and S Fahn), pp. 272-287. Butterworth, London.

Léger JM, Girault JA and Bolgert F (1987) Deux cas de dystonie isolée d'un membre supérieur inaugurant une maladie de Steele-Richardson-Olszewski. Revue Neurologique, 143, 140-142.

Maher ER and Lees AJ (1986) The clinical features and natural history of the Steele-Richardson-Olszewski syndrome (progressive supranuclear palsy). Neurology, 36, 1005-1008.

Mastaglia FL and Grainger KMR (1975) Internuclear ophthalmoplegia in progressive supranuclear palsy. Journal of the Neurological Sciences, 25, 303-308.

Miller BL, Lesser IM, Boone EB et al. (1991) Brain lesions and cognitive function in late-life psychosis. British Journal of Psychiatry, 158, 76-82.

Murray RM, O'Callaghan E, Castle DJ and Lewis SW (1992) A neurodevelopmental approach to the classification of schizophrenia. Schizophrenia Bulletin, 18, 319-332.

Nuwer MR (1981) Progressive supranuclear palsy despite normal eye movements. Archives of Neurology, 38, 784.

Ostergaard Kristensen M (1985) Progressive supranuclear palsy-20 years later. Acta Neurologica Scandinavica, 71, 177-189.

Perkin GD, Lees AJ, Stern GM and Kocen RS (1978) Problems in the diagnosis of progressive supranuclear palsy (SteeleRichardson-Olszewski syndrome). Canadian Journal of Neurological Sciences, 5, 167-173.
Rafal RD and Friedman JH (1987) Limb dystonia in progressive supranuclear palsy. Neurology, 37, 1546-1549.

Rogers D (1986) Bradyphrenia in parkinsonism: a historical review. Psychological Medicine, 16, 257-265.

Shimizu T, Inami Y, Sugita Y et al. (1990) REM sleep without muscle atonia (stage 1-REM) and its relation to delirious behavior during sleep in patients with degenerative diseases involving the brain stem. Japanese Journal of Psychiatry and Neurology, 44, 681-692.

Steele J, Richardson JC and Olszewski J (1964) Progressive supranuclear palsy. Archives of Neurology, 10, 333-359.

Takahashi H, Takeda S, Ikuta F and Homma Y (1987) Progressive supranuclear palsy with limbic system involvement: report of a case with ultrastructural investigation of neurofibrillary tangles in various locations. Clinical Neuropathology, 6, 271-276.

Thompson PD and Marsden CD (1992) Corticobasal degeneration. Ballière's Clinical Neurology, 1, 677-686.

Verhaart WJC (1958) Degeneration of the brain stem reticular formation, other parts of the brain stem and the cerebellum. An example of heterogenous systemic degeneration of the central nervous system. Journal of Neuropathology and Experimental Neurology, 17, 382-391.

World Health Organization (1992) The ICD-10 Classification of Mental and Behavioural Disorders: Clinical Descriptions and Diagnostic Guidelines. World Health Organization, Geneva.

(Received 30 September 1993; accepted 18 October 1993) 


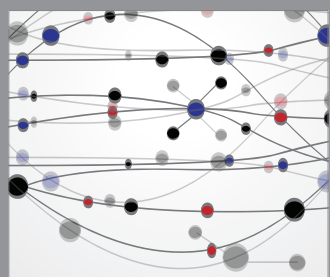

The Scientific World Journal
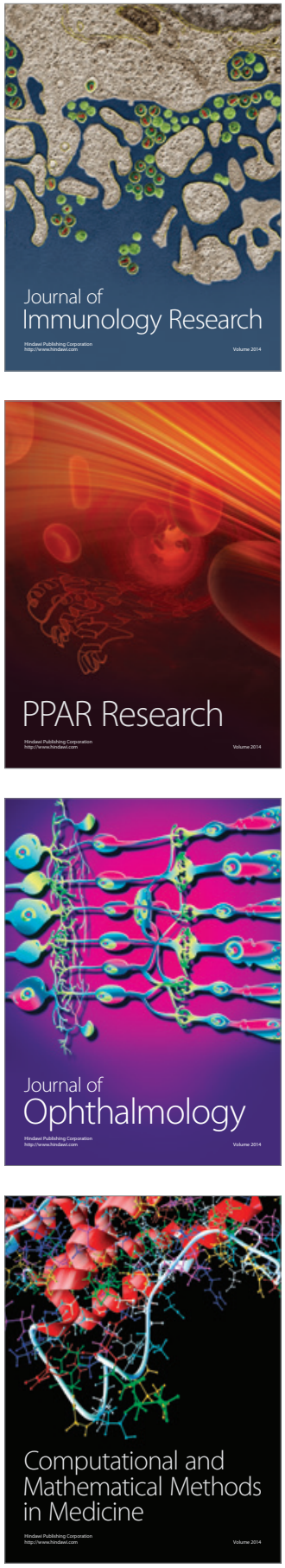

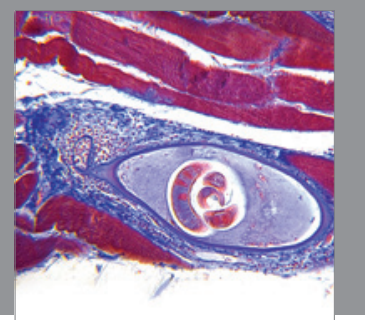

Gastroenterology

Research and Practice
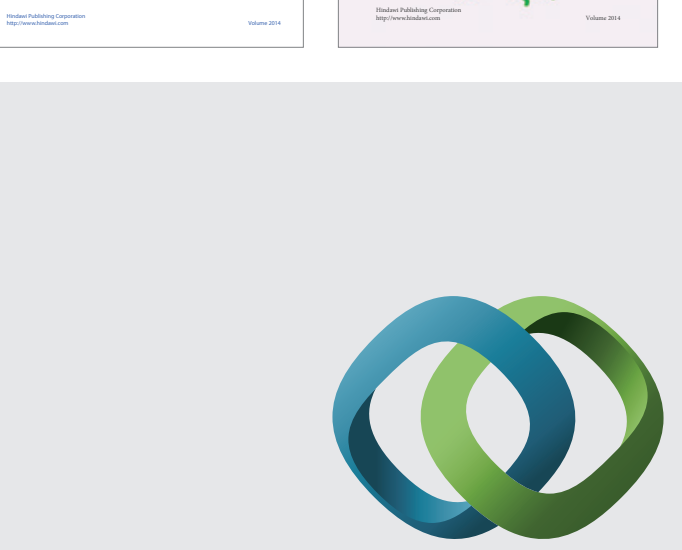

\section{Hindawi}

Submit your manuscripts at

http://www.hindawi.com
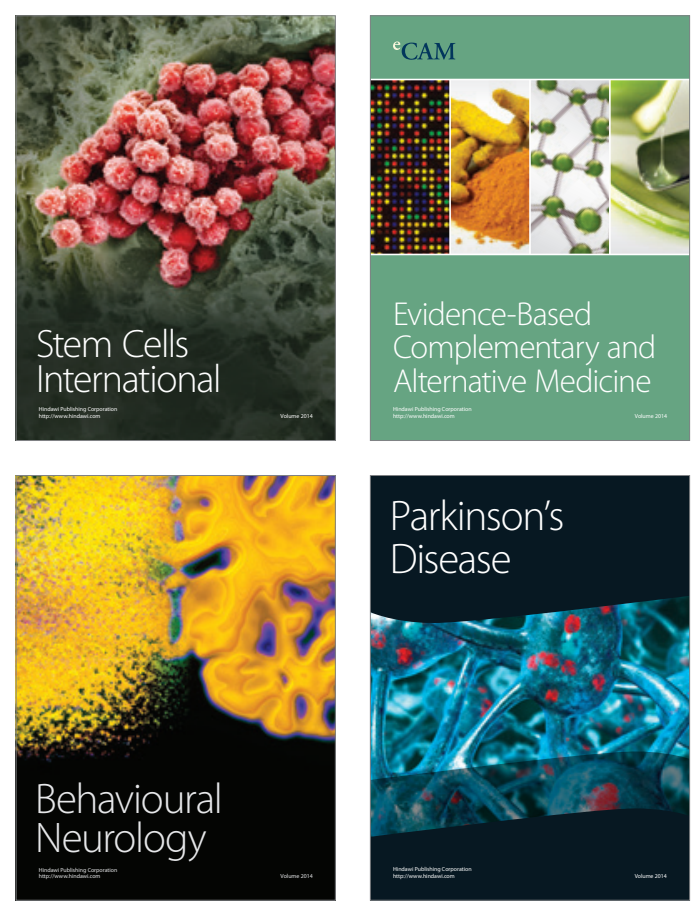

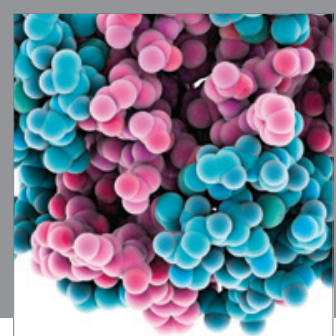

Journal of
Diabetes Research

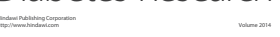

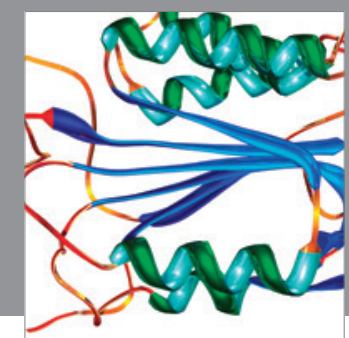

Disease Markers
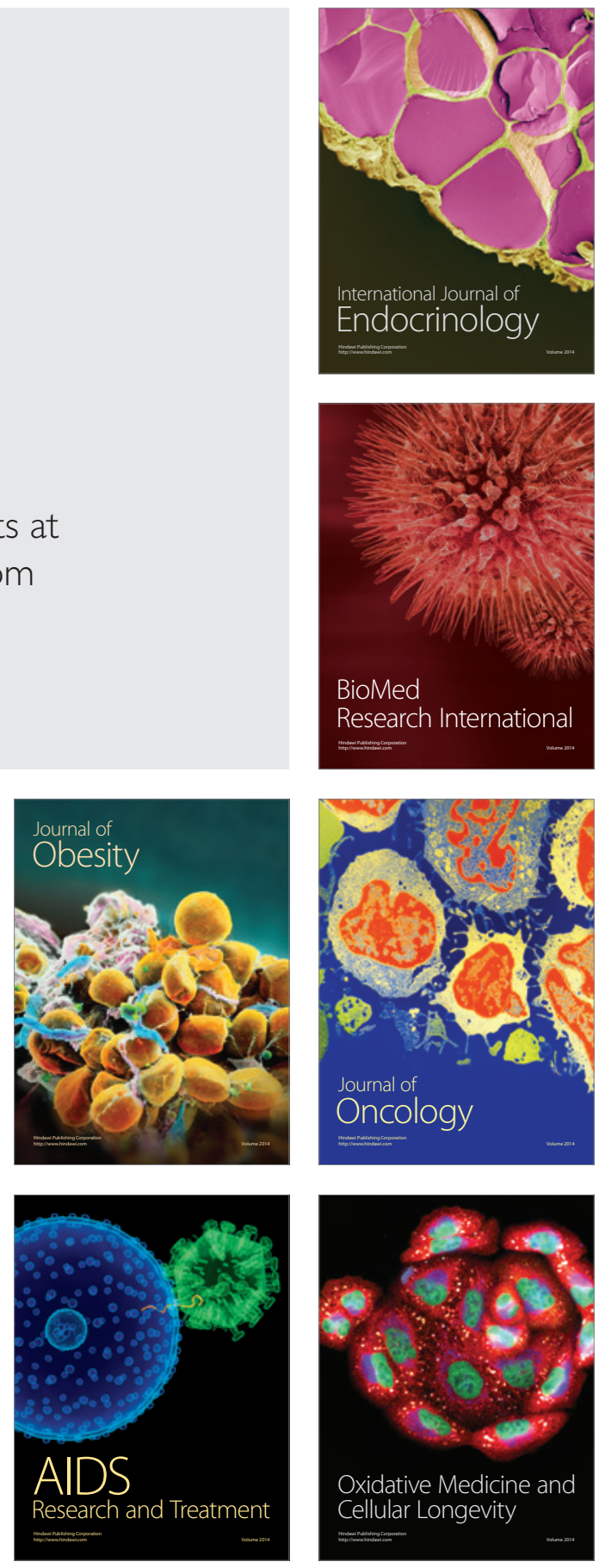